\title{
"gRpas", a Tool for Performance Testing and Analysis
}

\author{
Laurentiu Cucos and Elise de Doncker \\ Western Michigan University \\ \{lcucos, elise\}@cs.wmich.edu \\ http://aegis.cs.wmich.edu/ lcucos, \\ http://www.cs.wmich.edu/ elise
}

\begin{abstract}
This paper presents "gRpas", a tool written in Java and designed to help analyzing test results from scientific computing applications. "gRpas" stands for "gather Results / plot, analyze, and store". As one of its main features, the tool is easy to interface with the user program. Furthermore it provides for one click data filtering and plot generation, effective graphical display of program output, and statistical report generation on algorithm results and performance. gRpas also has built-in functionality for comparison testing between two or more algorithms or algorithm versions. We will present examples of its use with parallel multivariate integration routines. However, its target applications cover a wide class of scientific computing programs.
\end{abstract}

\section{Introduction}

Software applications in general and scientific computing programs in particular can be seen as multidimensional functions: $f: R^{n} \rightarrow R^{m}$. A program can take an $n$-variate input, and generate an $m$-variate output. To analyze the program behavior, the investigator selects a set of input values and compares the output with results obtained using a different method. The complexity of evaluating the program arises through a number of factors including: the problem to be solved and its parameters, the algorithm used and its parameters, random factors, total number of processors involved, etc.

For a given problem, some algorithms perform better than others, while for a given algorithm some problems will be solved more efficiently. Efficiency related results are generally affected by the problem parameters and the algorithm parameters.

The random factor is related to the fact that some algorithms require a set of random values. Ideally, the outcome must not depend on the random values; however this is not always the case. By repeating the same test a number of times, the developer is able to see if the output of the algorithm tends to the same value.

The number of processors involved can complicate the analysis. On the one hand, for each processor, the developer must monitor a number of efficiency 
related parameters; on the other hand, a disturbance of the distributed environment (such as in sharing bandwidth with another program) can possibly affect the outcome, introducing a random factor.

For those problems for which there are no proved solutions (either because they are too hard and require approximations - as for NP complete problems, or because they involve too many extra conditions - as the parallel processing, adaptive numerical integration, etc.), the development cycle often iterates as follows:

a) find a new algorithm (or start from a current version);

b) perform a set of tests;

c) based on results analysis improve the algorithm.

For the later stages, some common questions are: How much testing must be done to obtain significant results? Is the new algorithm better and how much better? Despite the fact that there are no practical limits on the amount of data that can be collected from the tests, it is desired to perform as few tests as possible (to save CPU cycles, or decrease development time).

Tests are usually automated by varying one input parameter while keeping the others constant. If, for the $i^{\text {th }}$ parameter, $k_{i}$ different values are tested, then a problem with $n$ input parameters and $\mathrm{m}$ output values generates $m \prod_{1 \leq i \leq n} k_{i}$ numbers to analyze.

Often, to evaluate improvements, the same set of tests are performed for different versions. In 1, Hooker mentions two types of algorithm comparisons: (i) competitive testing, and (ii) controlled experimentation. Competitive testing is more suitable for development, and tells which algorithm is faster but not why, whereas controlled experimentation is suitable for research and gives insight on how the code behaves under certain conditions. We can relate this classification with the following: (i) compare results from two or more versions. (ii) compare results from the same algorithm version;

For comparing algorithms in (i), it is indicated to examine the difference in result vectors from different algorithms for corresponding input parameters. There is often a trade-off between the amount of work done and the quality of the result. Algorithm A may be pessimistic and B more optimistic, so that requesting less accuracy from A may lead to results equivalent to B's. The performance profiles technique of 4 accounts for this characteristic in the comparison of $\mathrm{A}$ and B.

In (ii), the algorithm may be tested with respect to the influence of random factors, either due to the environment or a random component in the algorithm (such as through the use of random numbers). The difference between the (sample) result vectors in $m$-space is significant to measure performance dependence on the random factor(s). Their distance can be accounted for by using the mean and variance with respect to some or all of the output components.

Performance measures to test algorithm behavior as a function of varying input parameters are problem related. For example, "result" and "estimated 
error" output parameters can be analyzed as a function of input "tolerated error" by comparing the actual error (if known) and estimated error to the level tolerated. The situation is complicated by the fact that the dependence on different input parameters may be correlated, such as that of "actual error" on "tolerated error" and "allowed number of iterations". As another example, various scalability measures (as a function of the number of processors used) are discussed in [5].

Another issue to take into account is the stability of the algorithm. An unstable algorithm may cause a large change in results for a small change in the input parameters, or, magnify the small errors from earlier computation stages until the result deviates completely from the true answer. Examples can be found in recurrence relations, like in computing the power of $\phi=-\frac{1}{2}(\sqrt{5}+1)$ using the recursive formula $\phi^{n+1}=\phi^{n-1}-\phi^{n}$. Solving Fredholm equations of the first kind also belongs to this category 6].

In this paper we introduce the gRpas tool, designed to help analyze test results from scientific computing applications. The idea was originally motivated by applications in numerical integration. Section 2 below outlines the numerical integration framework that lead to the development of gRpas. Section 3 presents the tool's main characteristics, and Section 4 concludes the paper.

\section{Testing Numerical Integration Algorithms}

The two main ingredients of a numerical integration problem are: $I(\alpha)$ the integral, and $M(\beta)$ the integration method, where $\alpha$ and $\beta$ are various parameters. We denote by $I_{j}$ a problem $I\left(\alpha_{j}\right)$ and $M_{k}$ a method $M\left(\beta_{k}\right)$. We consider the integrand function, integration region, requested accuracy and maximum number of function evaluations as part of the integration parameters $\alpha$. A few examples of integration methods are: adaptive, Monte Carlo and quasi-Monte Carlo methods. Various cubature rules that can be used at the basis of adaptive multivariate integration methods are listed in [2].

A major issue in numerical integration is that often we don't know the characteristics of the integrand function. In general, we don't have answers to questions like: are there any singularities, how steep are they and where are they located?

We denote with $M_{j}^{o}$ the best algorithm that solves $I_{j}$, that is, the algorithm with the best convergence. Let $C(I, M)$ be the cost of solving $I$ using algorithm $M$, expressed in an appropriate measurement unit (e.g., number of function evaluations performed).

Given $M$, it is our goal to develop a set of tests to:

a) find $\beta_{k}$ such that on the average over all $I_{j}, C(I, M)$ is minimum. - Since it is impossible to handle all $I_{j}$, we need to determine a suitable test set for which we require that on the average $C(I, M)$ is minimized. Alternatively we may require that $M\left(\beta_{k}\right)=M_{j}^{o}$ for $I_{j}$ in the test set. Or, we may just want to extract as much information as possible about the algorithm behavior.

b) compare $M_{k}$ with other algorithms for the entire test space. 


\section{3 gRpas Tool}

gRpas [7] is a small application designed to help analyze results from scientific applications. Its main features are listed bellow.

\subsection{Flexible Application Interface}

For inter-operability and flexibility, all data is stored in an XML like format. For each input/output parameter, the user specifies a set of attributes: name, type, and tag. These are stored in a type repository and are used by the GUI module and re-used for similar tests. All results, along with input parameters, are stored in a tagged format.

\subsection{Option for Filtering or Combining Results}

Tests are performed in groups. Usually a set of tests is performed, analyzed and if there are satisfactory results, another group of tests is performed. If the results are not as expected, the code is changed and the same set of tests is repeated. gRpas can combine the results from different groups of tests or can present them individually. Figure 1 shows the tree structure: applications - versions - tests. In Figure 2 a Test Set is expanded to show the relation between Individual Tests and input parameters for an application with 4 parameters: $p_{1}, p_{2}, p_{3}, p_{4}$, where $p_{1}$ gets assigned three values and $p_{2}$ two. The Test Set is composed of 6 Individual Tests.

Note that although the version can be seen as an algorithm parameter, from the practical point of view it is better to consider it as a separate entity.

The user can filter certain results across multiple sets of tests for individual analysis, or can combine multiple tests from multiple sources.

\subsection{Flexible User Interface}

The application is mainly designed for algorithm comparisons, and is mainly intended for scientific software developers. The user interface is simple and is

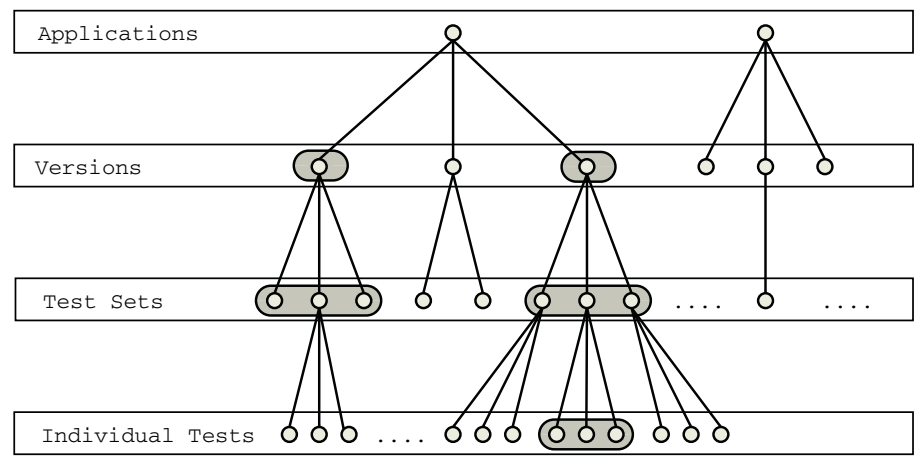

Fig. 1. Tests Tree Structure 


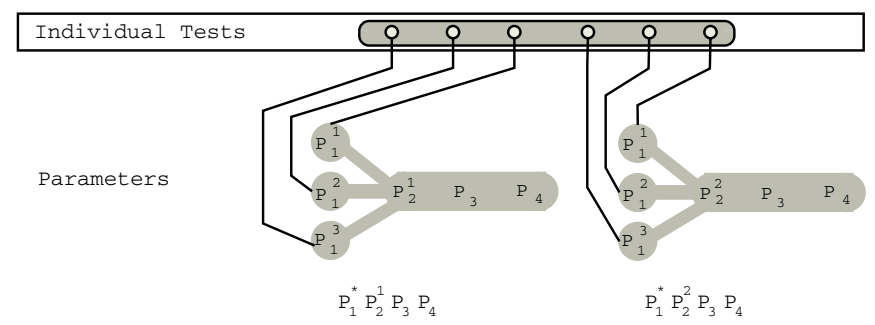

Fig. 2. Individual Tests and Parameters

point-and-click based. This allows for a short learning curve and fast response to the user. After data is collected, extended plots can be generated. Parameters defined in the input files can be selected by their names from drop-down menus. Multiple plots can be drawn in the same window and multiple windows can be displayed at once.

Figure $\mathrm{P}$ shows the main user interface. The table in the lower part lists all the Test Sets performed for a particular version of code (they correspond to the shaded areas in the Test Sets level in Figure 1 ). In the rightmost column the title shows the name of the code version, and the cells show the time when the

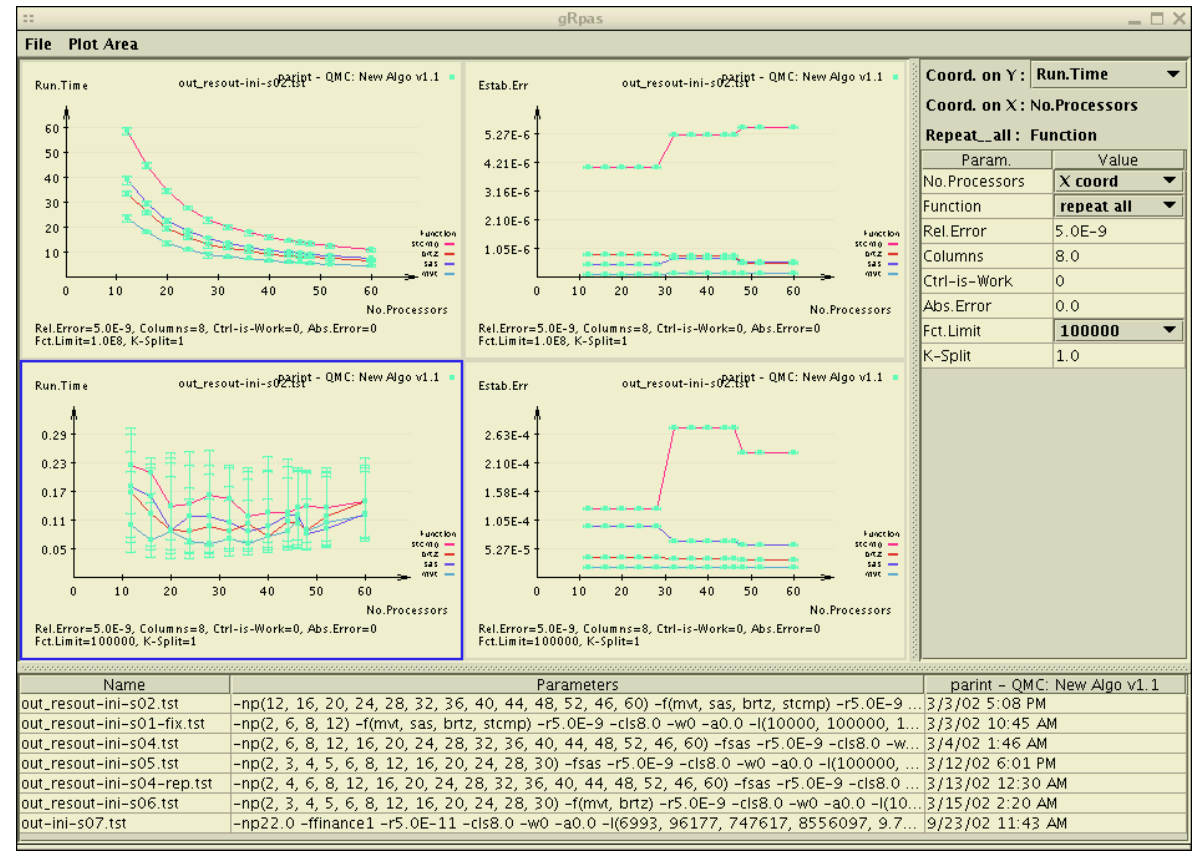

Fig. 3. gRpas: multiple plots, multiple window 
Sets were generated. The middle column shows the parameters used in the Set, and the left column shows the associated name for the Set.

Once the user has selected a Test Set, its parameters are shown in the control group situated in the right (they correspond to the type of area that is shaded in the Individual Tests level in Figure 1). This section has a double role: to show and to select the parameters to be plotted. In the top part the user selects the $\mathrm{Y}$ coordinate from a drop list containing all the output variables, while the $\mathrm{X}$ coordinate is selected from the input parameters with multiple values. Once the user has selected a parameter set, the plots are displayed in the center-left area.

The plots in Figure 1 graph results from solving multivariate integration problems using a quasi-Monte Carlo method with up to 60 processors. The variable input parameters are: Integrand Function, Number of Processors, and Function Limit. This particular Set consists of 325 Individual Tests, each repeated a number of times. On the left, the Run Time is plotted versus the Number of Processors for all the integrand functions using a different Function evaluation Limit, whereas on the right the obtained error is plotted. Each plotted point represents the median of multiple values. The data interval is shown as a closed segment.

Figure 4 presents two algorithm versions (note an additional column in the table Set area). The two versions have a number of identical Test Sets: Test s01, Test s02, Test s05, and Test s06 (which can be identified by the date in the algorithm version column). The plot shows the differences in the result values.

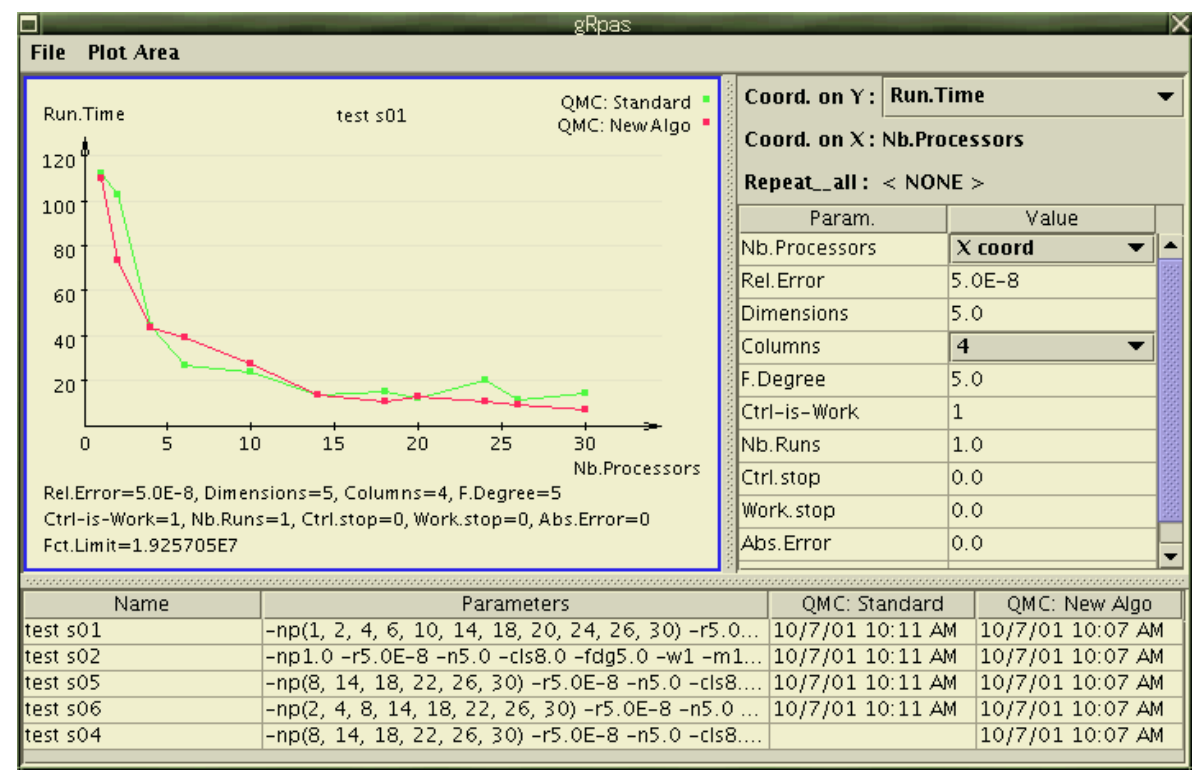

Fig. 4. gRpas: multiple algorithm plot 


\subsection{User Defined Plugins}

Extern modules can be linked to gRpas for additional functionality. The user can pre-process the output before displaying, or can link in a different application. The plugins must be written in java. The extern modules are called using menus. For example, Figure 5 shows the effect of applying the scalability plugin: for each test, the plot shows the inverse of the runtime multiplied with the runtime of the first test (which corresponds to the sequential run).

\subsection{Statistical Reports}

As mentioned earlier, the developer is interested in two types of comparisons. First, for a given algorithm $M$, what is the value of $\beta$ such that $M(\beta)$ gives the best performance for a set of problems $I_{j}$. Second, given two or more algorithms, which one performs better for the same set of problems.

Most of the time, a simple inspection of the plots gives enough information to continue analyzing the algorithm or to change it.

To generate more elaborate statistics we have two options, either implement a small set of functions in java as plugins, or export the data to applications like Splus or SAS. It is our goal to implement a set of functions that can perform comparisons at the click of a button.

For further consideration, we envision a new functionality for generating plots from non-common-parameters tests. Instead of performing Test Sets where some parameters are fixed while others vary, is possible to generate indicative plots

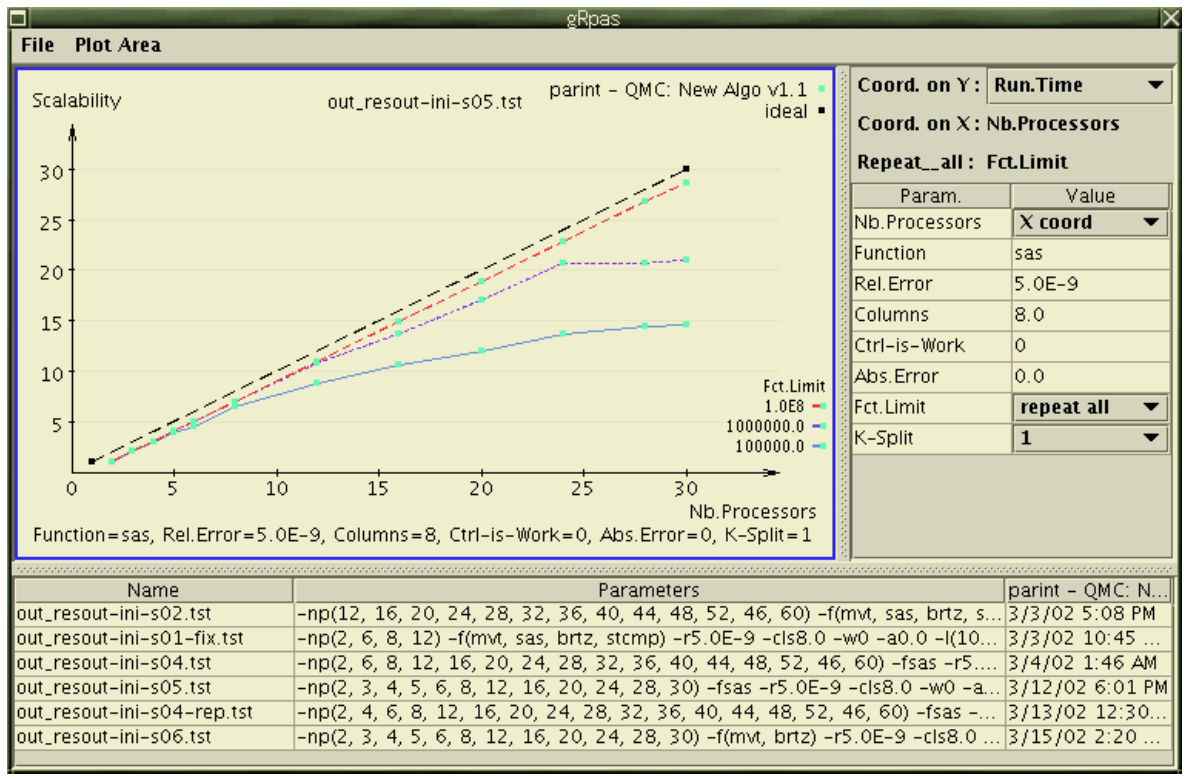

Fig. 5. gRpas: scalability plugin 
from Tests that don't share common parameters. In this way it is possible to reduce the number of performed tests, while increasing the problem investigation space.

\section{Conclusions}

In this paper we presented "gRpas", a tool to help analyze test results for scientific computing applications. Intended to be used for both competitive testing as well as controlled experimentation, the application has been extensively used in our parallel numerical integration research. We included samples of its usage from solving multivariate t-distribution (MVT) problems using a quasi-Monte Carlo method with up to 60 processors. The "gRpas" application and code is available for download at [7. It can also be executed online as an applet at 7].

\section{Acknowledgements}

The authors thank Krishna K, and Brendon Thiede for important programming contributions.

\section{References}

1. J. N. Hooker, "Testing heuristics: We have it all wrong", J. Heuristics, vol. 1, no. 1, pp. 33-42, 1995.

2. R. Cools. Monomial cubature rules since "Stroud": a compilation - part 2. J. Comput. Appl. Math., 112(1-2): pp. 21-27, 1999.

3. C. H. Chen, S. D. Wu, L. Dai, "Ordinal Comparisons of Heuristics Algorithms Using Stochastic Optimization", IEEE Transactions on Robotics and Automation, vol. 15, no. 1 , pp. 44-56, 1999.

4. J. N. Lyness and J. J. Kaganove, "A Technique for Comparing Automatic Quadrature Routines", The Computer Journal, vol. 20, no. 2, pp. 170-177, 1977.

5. R. Zanny, K. Kaugars and E. de Doncker, "Scalability of Branch-and-Bound and Adaptive Integration", in Proceedings of the Conference on Parallel Distributed Processing Techniques and Applications (PDPTA'01), pp. 674-680, 2001.

6. Press, W. H., Teukolsky, S. A., Vetterling, W. T. and Flannery, B. P, "Numerical Recipes in C - The Art of Scientific Computing", Second Edition, Cambridge University Press",1997.

7. "gRpas", http://aegis.cs.wmich.edu/\%7Elcucos/prjs/grpas/doc/gRpas.html 